\title{
De Fin de saison à Notre Ascendance, itinéraire génétique d'un poème du Mauricien René Noyau
}

\section{Robert Furlong et Gérard Noyau}

\section{OpenEdition}

\section{Journals}

Édition électronique

URL : https://journals.openedition.org/coma/3677

DOI : $10.4000 /$ coma.3677

ISSN : 2275-1742

\section{Éditeur}

Institut des textes \& manuscrits modernes (ITEM)

\section{Référence électronique}

Robert Furlong et Gérard Noyau, « De Fin de saison à Notre Ascendance, itinéraire génétique d'un poème du Mauricien René Noyau », Continents manuscrits [En ligne], 12 | 2019, mis en ligne le 15 février 2019, consulté le 14 janvier 2023. URL : http://journals.openedition.org/coma/3677 ; DOI : https://doi.org/10.4000/coma.3677

Ce document a été généré automatiquement le 14 janvier 2023.

\section{cc) $(1) \odot$}

Creative Commons - Attribution - Pas d'Utilisation Commerciale - Pas de Modification 4.0 International - CC BY-NC-ND 4.0

https://creativecommons.org/licenses/by-nc-nd/4.0/ 


\title{
De Fin de saison à Notre Ascendance, itinéraire génétique d'un poème du Mauricien René Noyau
}

\author{
Robert Furlong et Gérard Noyau
}

\section{René Noyau, écrivain mauricien}

1 René Noyau (1912-1984) partiellement africaine, se lance dans l'écriture à l'île Maurice à la fin des années 1920 et il écrit inlassablement jusqu'à sa mort en septembre 1984. Tout au long des 57 ans de sa vie d'écrivain, il éprouve un besoin insatiable d'écrire pour «briser les chaînes » que celles-ci soient imposées par la société ou par la politique. Son œuvre est à la fois d'une ampleur et d'une qualité incontestables. Il touche à tous les genres (poésie, nouvelle, roman, essai, aphorisme, théâtre, chroniques de presse...) en fonction des thèmes abordés : l'art, la politique, la négritude, l'amour, l'amitié, l'exploitation des démunis. René Noyau est le premier auteur mauricien à clamer son africanité et ce, avant le poète mauricien Édouard Maunick qui, lui, fera du métissage sa thématique principale. Mais si la voix de ce dernier, déclenchée depuis Paris, a bénéficié du soutien de la revue Présence Africaine et des personnalités littéraires qui l'animaient, celle de Noyau n'a eu que l'île comme espace d'expression sans grande résonance outremer.

2 Lorsqu'il se lance dans ses écrits africains (appellation choisie par lui) à partir de 1955, René Noyau a déjà de l'expérience en littérature. En 1934, il lance le surréalisme à Maurice avec son recueil L'Ange aux pieds d'airain (1934), publie des nouvelles teintées de surréalisme en 1936 avec Passerelles, puis, de nouveau, des poèmes avec Le Labyrinthe illuminé, avant la publication d'aphorismes en 1942 sous le titre Le Poinçon de cristal. Ses titres sont toujours construits sur des paradoxes comme si l'île Maurice et la vie qui s'y déploie n'étaient qu'un paradoxe. De retour d'un séjour en Europe, il réajuste progressivement son mode de vie et s'oriente vers de nouvelles thématiques: la réflexion politique ${ }^{2}$ et la revalorisation de son héritage africain, un choix courageux 
d'authenticité dans cette île attachant alors peu d'intérêt à la culture africaine. De ces écrits africains, le plus connu est le poème Séga de liberté de 1959, maintes fois cité et publié comme, par exemple, en 2016, dans la revue célébrant le $180^{\mathrm{e}}$ anniversaire de l'abolition de l'esclavage à Maurice ${ }^{3}$.

3 Ses écrits ont paru, dans un premier temps, sous des pseudonymes divers et nombreux, puis sous celui qui devint le plus fréquent pour ses nombreuses chroniques de presse et l'ensemble de sa poésie d'avant 1971, à savoir Jean Erenne... le nom Erenne venant de la vocalisation de ses initiales R. et N. Parmi ses écrits africains on retrouve

- en 1955 : Terre en feu ${ }^{4}$, poème ;

- en 1956 : Présence Africaine à l'île Maurice, essai ;

- en 1959 : Séga de liberté, poème ;

- en 1965 et 1968 : Les amis du peuple veillent, poème politique (premier de ce genre à Maurice) ;

- en 1968, année de l'indépendance mauricienne : Le Complexe de la peur, essai politique ;

- en 1971, Tention Caïma, le premier texte post-indépendance en kreol mauricien avec une version en français : Il y a toujours des caïmans...

Suivent en 1973 La Race d'Abel, pièce en prose retraçant une révolution pacifique par le peuple, et, en 1975, sous le titre La Race d'Abel - première partie, un poème dramatique racontant l'odyssée d'un homme ordinaire dans des pays étrangers. L'œuvre quasi intégrale de René Noyau a fait l'objet d'une publication en quatre volumes ${ }^{5}$ en 2012 et 2013. Cependant, de nombreux manuscrits restent à être publiés.

Image de couverture, René Noyau, L'œuvre , vol. I
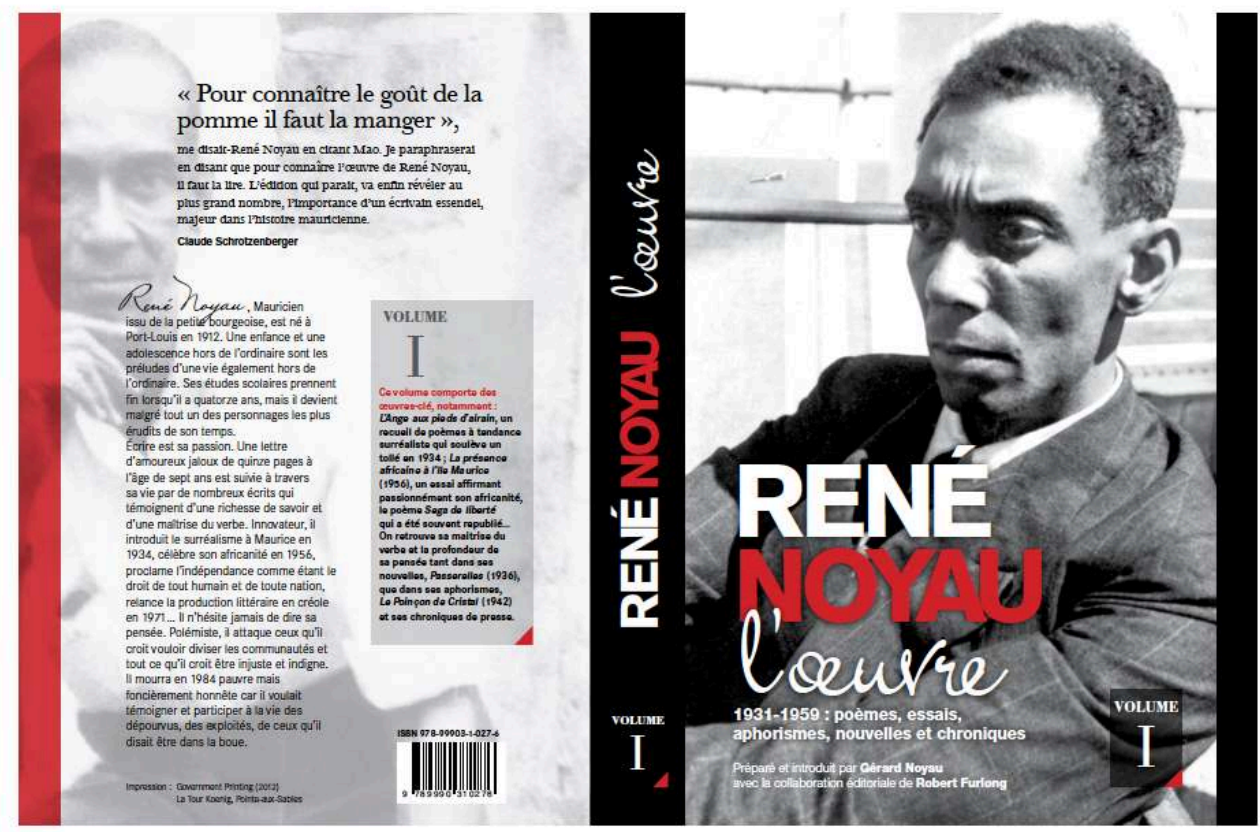

\section{Notre ascendance, un poème aux différentes versions}

5 À partir du milieu des années 1950, René Noyau - écrivant sous son nom ainsi que sous plusieurs pseudonymes dont le plus connu est Jean Erenne - se lance dans ce qu'il appelle ses «écrits africains ». Son œuvre littéraire est alors déjà bien établie: ses 
poèmes surréalistes publiés dans les recueils L'Ange aux pieds d'airain (1934) et Le Labyrinthe illuminé (1939) ont attiré l'attention sur lui et ont même été à la base de polémiques. En effet, comme il l'affirmera dans une causerie radiodiffusée en 1970, son ouverture aux tendances littéraires nouvelles avait choqué car « à Maurice, [...] il fallait que l'on restât rivé au classicisme, cramponné au romantisme ou agenouillé devant le Parnasse [et que] du symbolisme on en parlait à peine ${ }^{6} »$. Il partageait la conviction avec, notamment, Marcel $\mathrm{Cabon}^{7}$, son ami journaliste et poète, que la créativité mauricienne en matière artistique et littéraire était prisonnière de canons vétustes leur credo : la nécessité de «briser les moules étroits qui façonnaient la vie sociale et politique du pays" ajoutant: "nous nous sentions élus pour être à la tête de ce combat " ${ }^{8}$. René Noyau utilise volontiers le terme de "guérilla» pour qualifier son action menée avec quelques contemporains des années 1930. Cette action ira, par exemple, jusqu'à piéger, puis dénoncer publiquement une importante société littéraire locale en participant, sous des pseudonymes ronflants, à son concours littéraire annuel. Cette participation, avec des poèmes créés de toutes pièces à partir de vers empruntés à d'illustres poètes, avait retenu l'attention du jury qui les publiera dans la revue de cette société littéraire, L'Essor, sans se rendre compte de la supercherie... Son recueil de contes intitulé Passerelles (1936) aura le même impact en raison de ses caractéristiques peu conventionnelles.

6 Le souci d'africanité, qui devient progressivement une thématique essentielle de son œuvre, devait déjà couver en lui car il a promptement atteint son point d'éclosion lors de sa rencontre en 1950 à Paris avec Léopold Sedar Senghor, alors député français. Racontant ce moment d'exception vingt-trois ans plus tard dans le quotidien Le Mauricien', René Noyau évoque leur entretien au domicile parisien de Senghor. Au menu : la littérature en général et nègre en particulier, la place des Noirs à Maurice... Il $\mathrm{y}$ eut, enfin, ce moment très particulier où le fils de Senghor, âgé de 5 ou 6 ans alors, lui "donna un baiser sur la bouche", et Noyau d'ajouter: "ce baiser sur ma bouche de métis réveilla l'homme noir en moi ». De retour à Maurice après ce séjour européen, qui l'emmena aussi en Angleterre à la rencontre d'artistes et de peintres connus, René Noyau allait entrer dans une nouvelle phase de son œuvre et développer sa recherche intérieure autour de l'africanité mauricienne, comme si ce baiser d'enfant avait inoculé en lui l'impérieux devoir de révéler et valoriser ce que l'on pourrait appeler la négritude mauricienne, alors méprisée car toujours considérée comme servile même si l'esclavage avait été aboli une centaine d'années plus tôt. Dans cette île au passé esclavagiste, la population est alors composée de Blancs descendants de colons français, de Métis se sentant plus proches de leur part européenne, de Noirs issus d'esclaves, d'une majorité d'Indiens venus de la grande péninsule pour travailler dans les champs en remplacement de la main d'œuvre esclave affranchie et d'une poignée de Chinois tenant des boutiques d'alimentation. L'intelligentsia est majoritairement blanche et métisse et, parmi les littéraires, on ne trouve aucun écrivain ou poète qui soit noir de peau. Enfin, il faut préciser que peu de lettrés s'intéressent aux domaines littéraires et artistiques : « Pour $90 \%$ des Mauriciens, la littérature et l'art ne mènent pas au bazar, et l'écrivain et l'artiste, courbant sous leur vocation, passent leur chemin et on les regarde sans intérêt ${ }^{10} »$ dit René Noyau.

7 Parmi les textes les plus significatifs de Noyau en relation avec sa négrité, il convient de retenir un texte intitulé Notre ascendance publié dans le quotidien indépendantiste mauricien Advance le 9 juillet 1960. Ce texte est probablement resté sans écho depuis sa 
parution il y a presque 60 ans, mais la découverte de quelques manuscrits - dans une des deux malles où avaient été rangés, après le décès de René Noyau des manuscrits, des tapuscrits, des cahiers comprenant diverses notes, des journaux - révèle que ce poème a une histoire, qu'il est le fruit d'un travail assidu de corrections dont les différentes étapes sont disponibles. Originellement intitulé Fin de Saison, le poète a transformé le premier jet manuscrit en un texte toujours manuscrit sous le titre Fin de Cession avant qu'il ne soit dactylographié sous le titre Notre descendance, puis remanié de nouveau et, enfin, publié sous le titre Notre ascendance.

Premier jet du poème : Fin de Saison
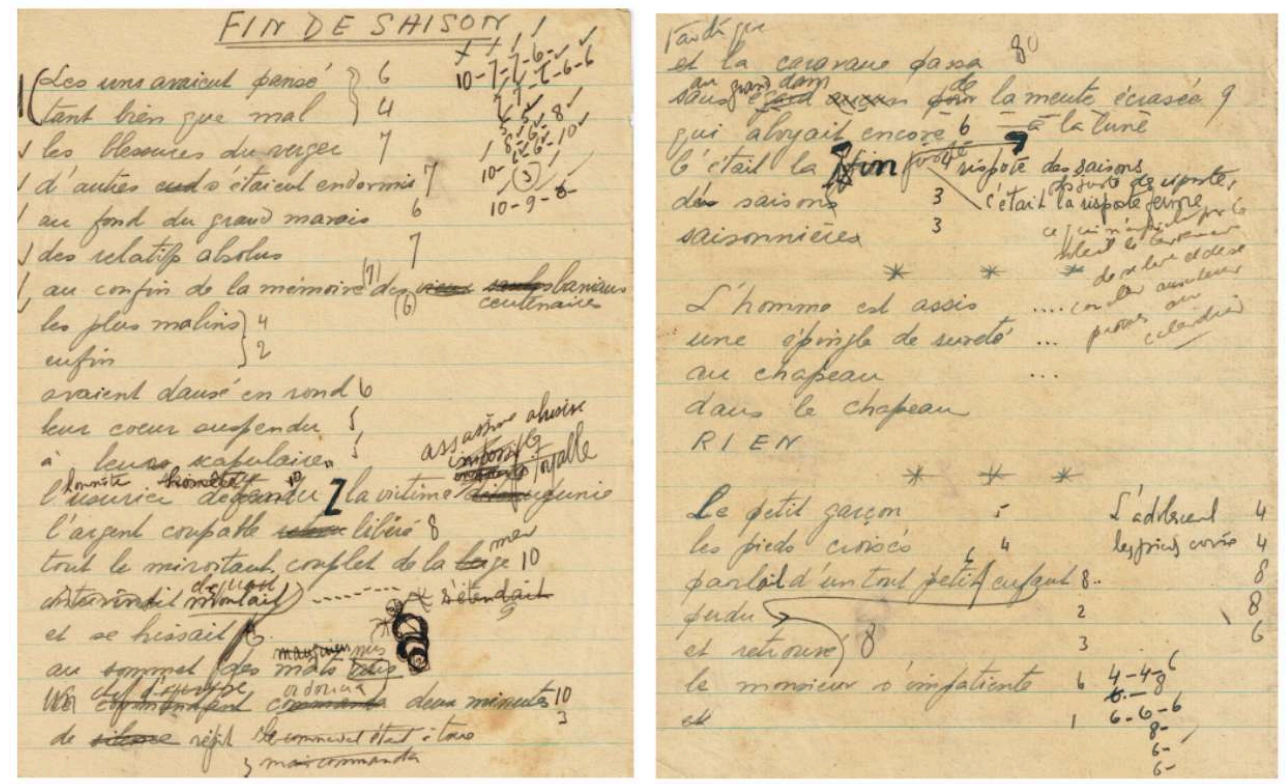

Collection particulière 


\section{Version 2 du poème : Fin de Cession}

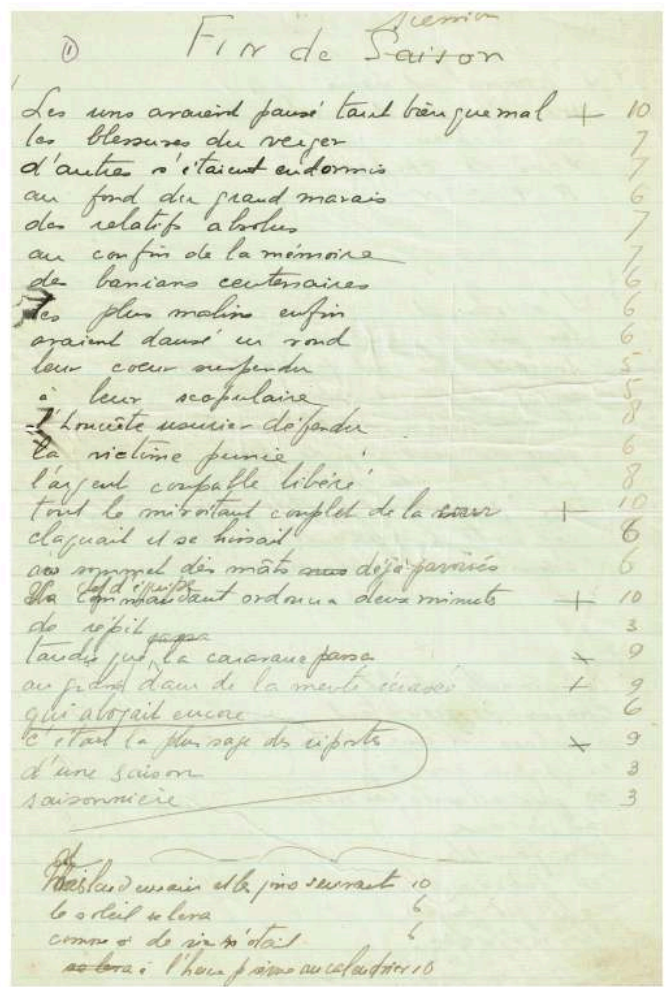

Collection particulière

\section{Notre transcription des versions 1 et 2}

\begin{tabular}{|c|c|}
\hline 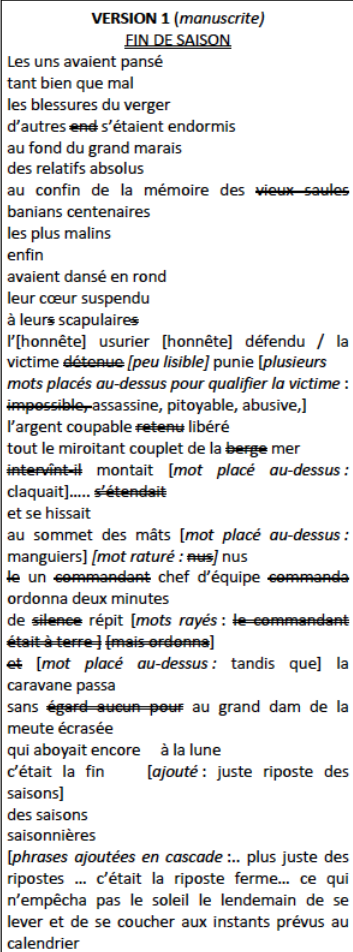 & 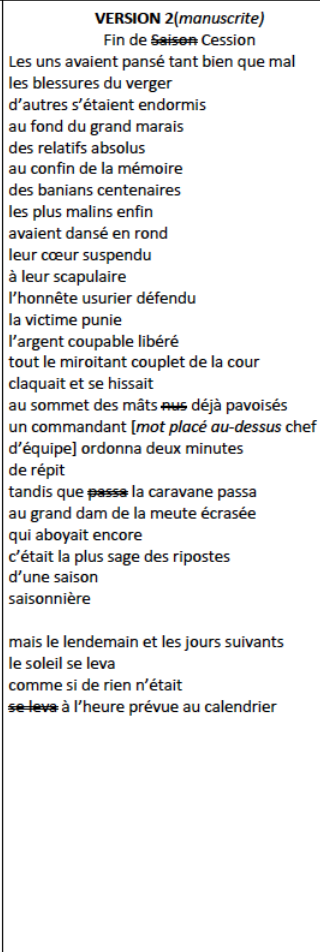 \\
\hline
\end{tabular}


Version 3 du poème : Notre descendance

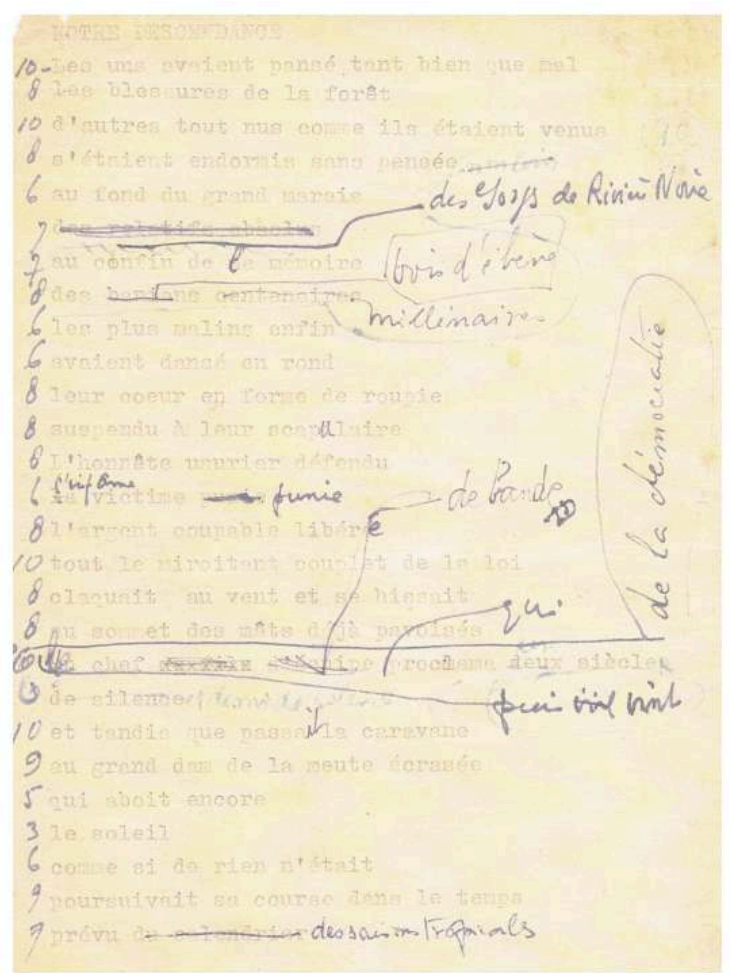

Collection particulière

\section{Version 4 du poème : Notre ascendance}

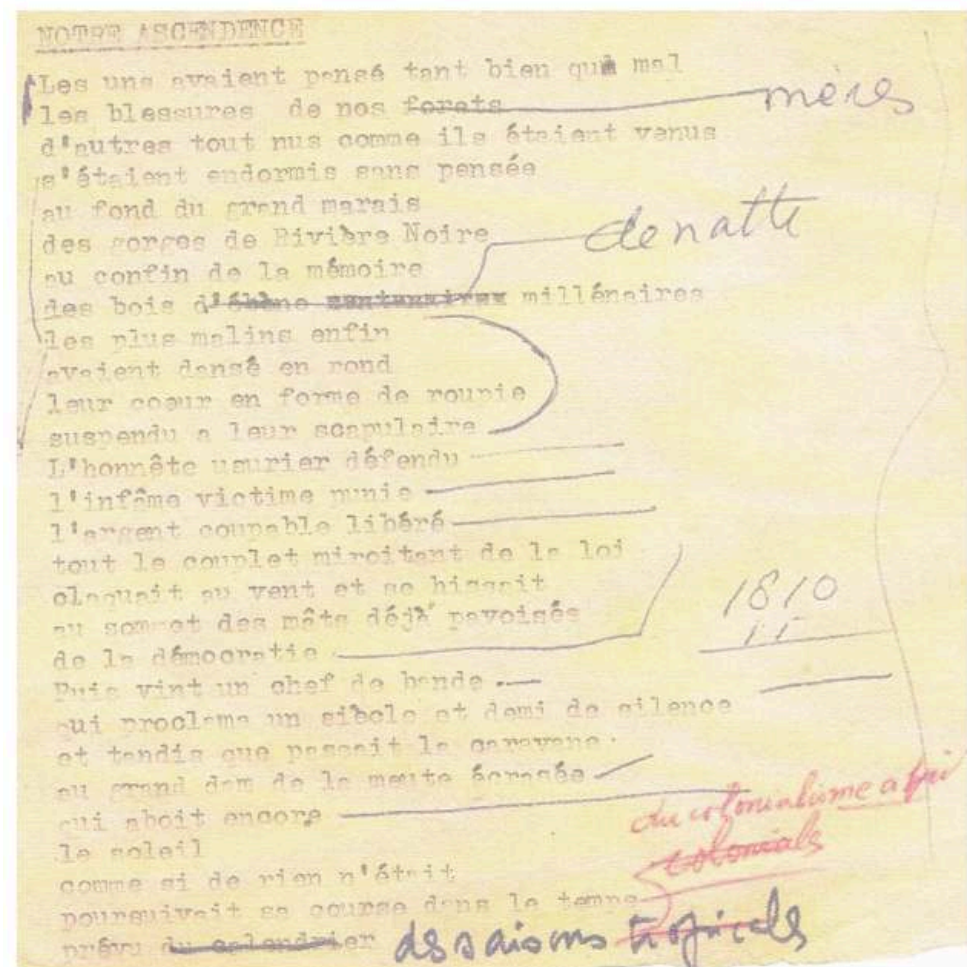

Collection particulière 
Notre transcription des versions 3 et 4

\begin{tabular}{|c|c|}
\hline 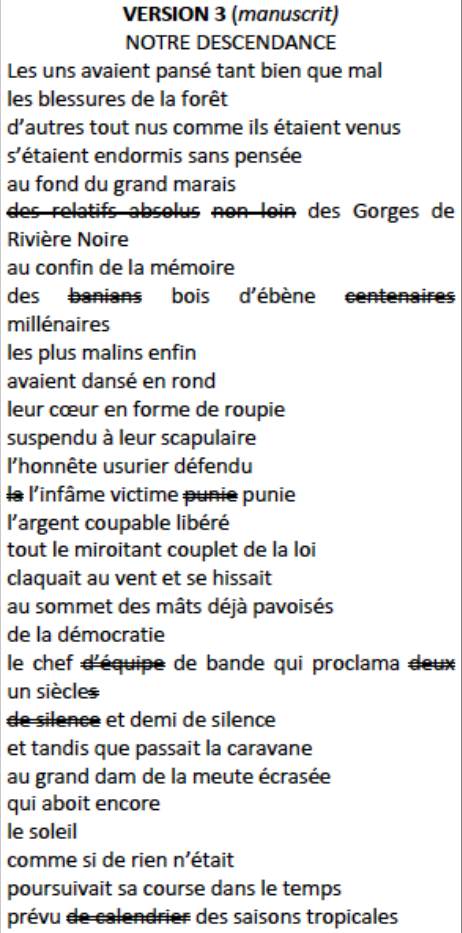 & $\begin{array}{l}\text { VERSION } 4 \text { (tapuscrit, corrigé à la main) } \\
\text { Notre ascendance } \\
\text { Les uns avaient pansé tant bien que mal } \\
\text { les blessures de nos mères } \\
\text { d'autres tout nus comme ils étaient venus } \\
\text { s'étaient endormis sans pensée } \\
\text { au fond du grand marais } \\
\text { des gorges de Rivière Noire } \\
\text { au confin de la mémoire } \\
\text { des bois de natte } \\
\text { millénaires } \\
\text { Les plus malins enfin } \\
\text { avaient dansé en rond } \\
\text { leur cœur en forme de roupie } \\
\text { suspendu à leur scapulaire } \\
\text { L'honnête usurier défendu } \\
\text { l'infâme victime punie } \\
\text { l'argent coupable libéré } \\
\text { tout le couplet miroitant de la loi } \\
\text { claquait au vent et se hissait } \\
\text { au sommet des mâts déjà pavoisés } \\
\text { de la démocratie } 1810 \\
\text { Puis vint un chef de bande } \\
\text { qui proclama un siècle et demi de silence } \\
\text { et tandis que passait la caravane } \\
\text { au grand dam de la meute écrasée } \\
\text { qui aboit encore [ajouté : du colonialisme a fui] } \\
\text { le soleil } \\
\text { comme si de rien n'était } \\
\text { poursuivait sa course dans le temps } \\
\text { prévu du du colonialisme abject } \\
\text { alsons }\end{array}$ \\
\hline
\end{tabular}

\section{Dernière étape du manuscrit publié}

\section{Notre ascendance}

Lies uns araient pansé tant bien que mal les blessures de nos mères

diautres tout nus comme ils étaient venus

s'étaient endormis sans pensée

au fond du grand marais

des gorges de Rivière Noire

- aux confins de la mémoire

des bois de nattes millénaires

Les plus malins enfin

avaient dansé en rond

leur coeur en forme de roupie

suspendu de leur scapulaire

Lihométe usurier défendu

l'infâme victime punie

l'argent coupable libéré

tout le couplet miroitant de la loi

claquait au vent et se hissait

au sommet des mâts déjà pavoisés

de la démocratie

Pris vint un chef de bande

qui proclama un siècle et demi de silence

et tandis que passait la caravane

an grand dam de la meute écrasée

qui aboie eneore

le soleil

comme si de rien n'était

poursuivait sa course dans le temps

prévu des saisons tropicales

JEAN ERENNE

Collection particulière 
Disposer des étapes génétiques de ce poème permet dès lors de suivre la pensée de Noyau et de mieux comprendre sa posture poétique par rapport à ces pages douloureuses de l'histoire de Maurice à travers les changements modifiant progressivement le texte originel ainsi que les titres successifs proposés. La présentation synoptique des quatre versions ${ }^{11}$ précédant celle publiée en juillet 1960 révèle le travail attentif du poète sur les mots et leur portée...

\section{Thème central et évolution des titres}

9 Le thème central de ce texte constitue une thématique tout à fait nouvelle en littérature mauricienne. Jusqu'à l'avènement de René Noyau, la poésie mauricienne ne s'était jamais aventurée à interroger l'histoire des origines de la population que ce soit dans sa totalité, Mauriciens d'origine européenne, africaine, malgache, asiatique et métisse inclus, ou dans chacune de ses composantes... Chanter les oiseaux, les fleurs et les arcs-en-ciel semblait suffire. René Noyau, quant à lui, écrivain au franc-parler redoutable, veut frontalement esquisser le sort de la population mauricienne issue de l'esclavage.

10 L'évolution des titres, tout d'abord, témoigne d'abord du souci de trouver l'intitulé le plus juste. Le premier titre, Fin de saison, est plutôt pudique, même si, sur le manuscrit, le poète l'énonce fièrement, en lettres majuscules, doublement soulignées, voire ponctuées. Cette Fin de saison est celle marquant la fin de la « saison » d'esclavage que la puissance colonisatrice française avait entretenu dès la prise de possession de l'île en 1715 et à laquelle il a été mis fin 25 ans après le passage sous colonisation anglaise de 1810. La réflexion que ce premier jet suscite en Noyau et les corrections qu'il porte au texte originel l'amènent, dans sa deuxième version, à modifier également le titre et à le faire évoluer tout en prenant soin de rester proche des sons du mot «saison». En utilisant un symbole de correction familier aux typographes, il indique qu'il change le mot «saison " pour celui de « cession ». Ce choix de titre Fin de cession implique alors qu'il sera question de personnes ayant, en quelque sorte, retrouvé possession d'euxmêmes après un temps pendant lequel elles avaient été « cédées ». Mais la relecture de cette deuxième version amène Noyau à un autre choix: celui de s'impliquer personnellement dans la masse de personnes libérées et, même, à s'y donner un rôle... Il choisit alors un titre à la fois énigmatique et étrange : Notre descendance... Sa relecture lui fera comprendre le caractère inapproprié de ce titre, le poème ne parlant pas ici de générations futures, ce qui aurait justifié le terme "descendance", mais bien au contraire de générations passées, ancestrales... Le titre Notre ascendance s'impose alors et sera celui de la version publiée dans la presse le 9 juillet 1960 : Noyau revendique ainsi publiquement une ancestralité africaine, la première en littérature mauricienne.

\section{Version $^{12} 1$ : Fin de Saison}

11 La première version manuscrite de ce texte, intitulé Fin de saison, se trouve sur deux pages dans un cahier d'écolier ${ }^{13}$ contenant des ébauches de plusieurs poèmes, la deuxième page comprenant deux autres poèmes, «L'homme est assis » et «Le petit garçon ». Les deux premiers vers seront à partir de la version 2 fondus en un seul « Les uns avaient pansé tant bien que mal » et ce vers demeurera le vers initial identique dans toutes les versions. Il en sera de même pour les vers 8 et 9 devenus un seul vers 
(" les plus malins enfin ») dès la $2^{\mathrm{e}}$ version et ce vers restera également le même dans toutes les versions. Au regard de la teinte de l'encre, ce texte de 26 lignes, en lettres cursives, semble avoir fait l'objet d'un premier jet sans hésitation ni arrêt dans sa rédaction. Le travail de correction et d'enrichissement dans une autre encre vient progressivement porter des modifications au vers 7 d'abord, puis à tous les vers à partir $\mathrm{du}$ vers 13. Sur le fond, on retrouve plusieurs exemples de l'humour noir caractéristique de René Noyau qui se manifeste à travers des affirmations paradoxales, proches de l'oxymore, telles que « des relatifs absolus », «l'honnête usurier » ou «la victime punie».

12 Le premier changement, au vers 7, porte sur les mots " vieux saules » qui sont biffés et remplacés par les mots «banians centenaires ». Fort probablement, les mots «vieux saules » ne convenaient plus au projet du poète car ils renvoient à un contexte végétal européen : les mots «banians ${ }^{14}$ centenaires » venus en remplacement évoquent mieux le contexte mauricien dans lequel se déroule l'évènement à la base du poème. Cet évènement, manifestement tragique, concerne une masse d'individus comprenant, d'une part, des blessés ayant "pansé " par eux-mêmes, donc sans soutien ni expertise médicale ("tant bien que mal »), des blessures et, d'autre part, ceux moins chanceux, " endormis » d'un sommeil possiblement éternel puisqu' " au fond du grand marais ». Entre les survivants et les morts relégués « au confin ${ }^{15}$ de la mémoire » se trouvent « les plus malins » qui survivent parce qu'accrochés à un talisman de nature religieuse, plus spécifiquement catholique : en l'occurrence, un scapulaire... Allusion probablement au baptême nécessaire des esclaves imposé par le Code Noir et à l'objet symbolique obtenu lors du sacrement catholique de la confirmation.

Dans le contexte de l'histoire de Maurice, il y eut deux types d'importation de maind'œuvre à grande échelle: celle des esclaves au cours du xvII siècle et celle des travailleurs engagés d'origine asiatique importés pour remplacer les esclaves dans les champs au lendemain de l'abolition de l'esclavage au cours du XIx et au début du Xx siècle. Les premiers furent majoritairement africains et malgaches, les seconds indiens. La référence au scapulaire et, dans des versions ultérieures, aux Gorges de Rivière Noire, lieu où se retrouvèrent nombre d'esclaves marron, confirme que les masses dont il est question dans un premier temps dans ce poème sont des masses d'esclaves. Les vers 13,14 et 15 se réfèrent aux bénéficiaires réels de l'abolition de l'esclavage: «l'honnête usurier » désignant les propriétaires d'esclaves qui furent généreusement indemnisés, «la victime punie » désignant l'esclave libéré sans aucune compensation, « l'argent coupable » étant cette masse considérable d'argent qu'obtinrent les anciens maîtres et avec lequel l'Angleterre s'achetait une bonne conscience.

René Noyau fait adroitement intervenir une pause temporelle ordonnée par un «chef d'équipe » ou "commandant» dans lequel on peut reconnaittre les premiers gouverneurs anglais, de Sir Robert T. Farquhar (1810-1820) à Sir William Nicolay (1833-1840) sous l'égide duquel fut proclamée l'abolition de l'esclavage et s'organisèrent les premières importations de laboureurs indiens: le vers «tout le miroitant couplet de la mer » y fait directement allusion, parce que l'engagement d'importer des travailleurs de l'Inde ainsi que les promesses d'indemnisation des propriétaires avaient été le «miroitant couplet » des Anglais que, métaphoriquement, la mer confirme en les amenant à bon port... Ces derniers arrivants constituent la deuxième masse convoyée par "caravanes" vers leurs lieux de campement et de travail « au grand dam de la meute écrasée » des anciens esclaves à qui rien n'avait été 
proposé : ni travail rémunéré, ni plan d'insertion. Fin d'une saison, donc: celle de l'esclavage, sans que cela ne perturbe en aucune façon les cycles naturels du temps, comme l'ajoute le poète en allongeant le poème par des lignes en marge.

\section{Version 2 et 3 : Fin de cession suivi de Notre descendance}

15 Le décor étant clairement campé et la trame référentielle historique précisée, les versions suivantes du poème seront autant d'occasions pour affiner certains mots, consolider certains propos. Ainsi, la version 2 sera une copie quasiment sans rature de la version 1 , compte tenu des corrections portées... «Tout le miroitant couplet de la mer » devient celui «de la cour », celle de la puissance ayant aboli l'esclavage et les " mâts nus » deviennent dans cette version des "mâts pavoisés » car l'abolition n'a finalement entraîné aucun désordre, ni de la part des affranchis, ni de celle des grands propriétaires.

16 Les modifications de la version 3 sont plus significatives. D'abord, le titre, en majuscules comme fut celui de la version 1, devient NOTRE DESCENDANCE, titre par lequel le poète s'implique personnellement comme partie prenante de ces faits historiques. Le grand marais, où reposaient les morts, est remplacé par une référence explicite aux " gorges de Rivière Noire ", lieu mythique où se réfugiaient les esclaves en fuite et proche de la montagne du Morne où des milliers se seraient jetés de la falaise pour ne pas se faire capturer. Les «banians » cèdent la place au «bois d'ébène » et « centenaires » est remplacé par " millénaires » : plus de confusion possible car il s'agit bien là explicitement d'esclaves que l'on appelait «bois d'ébène » au temps de la traite. Enfin, pour la première fois, le « commandant » de la version 1, toujours représentant symboliquement les nouveaux colonisateurs, devenu "chef d'équipe", est enfin nommé «chef de bande». Son action est maintenant qualifiée comme reliée «à la démocratie ", une démocratie très particulière car c'est en son nom qu'il sera demandé de faire silence et ce pendant « un siècle et demi »... soit de 1810 (date de l'arrivée des Anglais qui abolirent l'esclavage) à 1960 (date de ce poème revendiquant une relecture de ce passé), même si le cours naturel du temps « des saisons tropicales » n'en a guère été affecté.

\section{Version 4 : Notre ascendance et version finale publiée}

17 La version 4 est la dernière ébauche avant publication : on peut penser que le poète est alors satisfait de la tournure finale. Les nouvelles corrections ne sont pas innocentes. Une fois encore, le titre est modifié et passe de Notre descendance à Notre ascendance ${ }^{16}$, René Noyau réalisant soudain qu'il ne peut se situer aux origines de cette évolution, mais plutôt comme son dernier maillon. Les «blessures " que les uns soignent comme ils peuvent deviennent celles de "nos mères ", ces femmes victimes de tant d'abus et de méfaits au temps de l'esclavage. Le " bois d'ébène » change et un arbuste endémique, le «bois de natte ", prend sa place. Le « miroitant couplet de la cour » devient « le couplet miroitant de la loi », celle rendant obligatoire l'affranchissement des esclaves. Noyau ajoute, par ailleurs et pour la première fois une date en inscrivant 1810 comme date de l'avènement de la démocratie et qui est la date du passage de l'île sous autorité anglaise. Enfin, les saisons dites «tropicales» sont requalifiées en tant que « coloniales". 
Mais la version publiée, elle, ne retient pas les « saisons coloniales » qui restent, donc, tropicales. Et le « bois de natte » devient le bois de « nattes »... La date de 1810 disparaît aussi, en même temps qu'une référence explicite au colonialisme. Autre modification, qui relève plutôt de la correction de langue : le mot " confins ", au singulier dans toutes les versions antérieures, devient comme il se doit " confins » et le terme "aboit» des versions manuscrites 3 et 4 est enfin orthographié correctement : « aboie »...

\section{En guise de conclusion}

19 L'œuvre littéraire de René Noyau n'a pas encore fait l'objet d'analyses approfondies. En réalité, elle sort à peine de l'ombre dans lequel son auteur l'avait lui-même reléguée en se retirant des circuits d'édition quelques années après avoir publié à compte d'auteur un livre qui fera date dans l'histoire de la littérature mauricienne... En effet, en 1971, il publie en langue créole accompagnée d'une version en français un conte-fable intitulé Tention Caïman (Il y aura toujours des caïmans) contribuant ainsi à relancer la production littéraire en créole à Maurice, une production un moment coincée dans des débats idéologiques comme si elle avait du mal à intégrer la notion d'indépendance politique. Bien des textes de René Noyau sont encore à l'état de manuscrit ou de tapuscrit et restent à être un jour publiés. La question est de pouvoir développer un intérêt pour l'ensemble de son œuvre. Les quatre volumes publiés en 2012 sous le titre René Noyau, l'œuvre, constitue un ensemble présentant divers modes d'expression de cet auteur dont l'œuvre est ample, riche et féconde pouvant ainsi être partagé, étudié et analysé. La présente étude génétique est une esquisse de ce que l'on peut découvrir en étudiant les productions de cet écrivain particulièrement attentif au moindre de ses mots et aux inflexions sous-jacentes.

\section{NOTES}

1. La date exacte de la naissance de René Noyau reste une énigme. Plusieurs fois, il écrit qu'il est né le 6 novembre 1911 comme dans une lettre du 6 novembre 1978: "Le 6 nov. C'est mon anniversaire. » Dans le seul passeport que nous ayons, la date de naissance indiquée est le 14 avril 1912.

2. Notons les articles suivants publiés dans la presse: "Politique d'abord", "Jean-Jacques Rousseau ou De L'Indépendance, «L'île Maurice indépendante: La situation actuelle et nos tâches ».

3. 180 Anniversary of abolition of Slavery in Mauritius 1835-2015, Port-Louis, Le Morne Heritage Trust, 2016.

4. Terre en feu, mon premier poème africain, de René Noyau à Françoise Ligier le 31 mars 1978.

5. Gérard Noyau et Robert Furlong, René Noyau, l'œuvre (4 volumes), Port-Louis (Ile Maurice), Government Printing, 2012. Contenu général : vol. I : 1931-1959: poèmes, essais, aphorismes, nouvelles et chroniques ; vol. II : 1960-1978 : poèmes, essais, conte en créole et chroniques; vol. III : Inédits I : essais, théâtre et écrits surréalistes ; vol IV.: Inédits II : poèmes, pensées, contes, travaux inachevés, lettres, opinions. 
6. Extrait du premier volet d'une causerie radiophonique en trois parties intitulée Actualités mauriciennes en date du 10 avril 1970, reproduite dans René Noyau, l'œuvre, vol. III, p. 37-58.

7. Marcel Cabon (1912-1972), écrivain et journaliste, auteur de nombreux recueils de poèmes et de contes. Également créateur de plusieurs revues littéraires et rédacteur en chef d'importants quotidiens.

8. Marcel Cabon, «In Memoriam Gobin Rottoo », Advance, 9 juin 1970.

9. Le Mauricien, $1^{\mathrm{er}}$ mai 1973, René Noyau : "Ma rencontre avec Senghor », reproduit dans René Noyau, l'œuvre, vol. II, p. 405-408.

10. Extrait du deuxième volet de la causerie radiophonique déjà citée et diffusée le 17 juillet 1970.

11. Nous ne reprenons pas ici le décompte des syllabes figurant sur les manuscrits et tapuscrits d'origine car notre propos ici se cantonne à l'analyse thématique de ce poème et de ses versions. Ce décompte est inscrit à côté des vers sur les reproductions en appendice.

12. Voir la reproduction des manuscrits en appendices.

13. Gérard Noyau a retrouvé dans une malle léguée par son père René Noyau plus d'un millier de manuscrits et de tapuscrits. Environ 200 manuscrits et 250 tapuscrits sont à publier encore, dont 84 manuscrits et 96 tapuscrits en kreol.

14. Le banian (mot d'origine indienne) est un arbre originaire de l'Inde pouvant être gigantesque tant en hauteur qu'en circonférence, étant donné les excroissances aériennes qui pendent et prennent racine en touchant le sol. Dans le contexte mauricien, l'arbre banian est aussi appelé «multipliant» ou, en kreol, « pielafous ». Auparavant, dans les villages, cet arbre était celui sous lequel les vieux du village se rencontraient et racontaient parfois aux jeunes l'histoire des origines ou, tout simplement, des contes traditionnels édifiants.

15. Noyau gardera le mot confin orthographié sans « $s$ » jusqu'à la version finale publiée en juillet 1960.

16. La première utilisation de ce titre est mal orthographiée, Noyau tapant à la machine le mot ASCENDENCE...

\section{RÉSUMÉS}

Bien que l'écrivain mauricien René Noyau (1912-1984) soit méconnu, son œuvre ample et féconde appelle une grande attention. Il était un écrivain engagé et novateur, un pionnier de la liberté personnelle et littéraire, quels que soient l'inconfort et l'éventuel danger d'une telle posture. Ces caractéristiques se retrouvent dans le poème Notre Ascendance, dont la genèse est analysée dans l'étude qui suit.

René Noyau (1912-84), Mauritian writer, may lack wide recognition, but his extensive and rich work commands and repays scrupulous attention.He was a passionately committed and innovative man of letters, a pioneer of personal and literary freedom, however uncomfortable or dangerous that might have been. These characteristics can be found in the carefully crafted poem Notre ascendance, which is explored in the following study.

\section{INDEX}

Mots-clés : esclavage, négritude, négrité, littérature mauricienne 


\section{AUTEURS}

\section{ROBERT FURLONG}

ROBERT FURLONG se consacre depuis 2004 à la littérature mauricienne. Il a publié, entre autres, la biographie de Marie Leblanc (2005), une anthologie de la littérature en créole mauricien ( $L a$ Production littéraire mauricienne : la production créolophone, 2008), plusieurs inédits de Malcolm de Chazal, également En revues et en français. Une anthologie de nouvelles, chroniques et contes mauriciens, parue dans des revues littéraires locales en 2015, et des articles sur la dynamique littéraire mauricienne dans des revues spécialisées. Robert Furlong est Officier dans l'ordre des Arts et des Lettres.

\section{GÉRARD NOYAU}

GÉRARD NOYAU, pédagogue de carrière en Grande-Bretagne, est le fils de l'écrivain mauricien René Noyau. Il s'est mis, à partir de 2010, à inventorier et rassembler les écrits de son père dont une grande partie était inédite, aux fins de publication. Avec la collaboration éditoriale de Robert Furlong, il a publié en 2012 et 2013 quatre volumes intitulés René Noyau, L'œuvre (Port-Louis, Ile Maurice : Government Printing). Il travaille en ce moment sur la traduction en anglais des poèmes de son père pour une publication courant 2019. 